\title{
Laser spectroscopy of iridium monoboride
}

\author{
Jianjun Ye, H. F. Pang, A. M.-Y. Wong, J. W.-H. Leung, and A. S.-C. Cheung ${ }^{\text {a) }}$ \\ Department of Chemistry, The University of Hong Kong, Pokfulam Road, Hong Kong
}

(Received 30 August 2007; accepted 3 March 2008; published online 21 April 2008)

\begin{abstract}
High resolution laser induced fluorescence spectrum of IrB in the spectral region between 545 and $610 \mathrm{~nm}$ has been recorded and analyzed. Reacting laser-ablated iridium atoms with $1 \% \mathrm{~B}_{2} \mathrm{H}_{6}$ seeded in argon produced the $\mathrm{IrB}$ molecule. This is the first experimental observation of the $\mathrm{IrB}$ molecule. Four vibronic transition bands, $(v, 0)$ with $v=0-3$ of an electronic transition system, have been observed. Spectra of all four isotopic molecules, ${ }^{191} \operatorname{Ir}^{10} \mathrm{~B},{ }^{193}{ }^{110}{ }^{10} \mathrm{~B},{ }^{191} \operatorname{Ir}^{11} \mathrm{~B}$, and ${ }^{193}{ }^{1}{ }^{11} \mathrm{~B}$, were recorded. Isotopic relationships confirmed the carrier of the spectra and the vibrational quantum number assignment. Preliminary analysis of rotational lines showed that these vibronic bands are with $\Omega^{\prime}=2$ and $\Omega^{\prime \prime}=3$. The electronic transition identified is assigned as the $[16.5]^{3} \Pi_{2}-X^{3} \Delta_{3}$ system. Partially resolved hyperfine structure which conforms to the Hund's case $a_{\beta}$ coupling scheme has been observed and analyzed. The bond length $r_{0}$ of the lower $X^{3} \Delta_{3}$ state of $\operatorname{IrB}$ was determined to be $1.7675 \AA$ A. (C) 2008 American Institute of Physics. [DOI: 10.1063/1.2901964]
\end{abstract}

\section{INTRODUCTION}

Metal borides are refractory compounds of considerable interest in thin film coating because of their attractive surface properties and exceptional corrosion resistance. ${ }^{1}$ Many transition metal borides are known to be good catalysts for hydrogenation of alkenes and alkynes, reduction of nitrogenous functional groups, and deoxygenation reactions. ${ }^{2}$ In addition, it is recently known that solid magnesium diboride $\left(\mathrm{MgB}_{2}\right)$ is a superconductor at very low temperature. ${ }^{3}$ Despite these important characteristics, experimental study of metal boride compounds is still very limited. In the past few years, some efforts have been made to determine the spectroscopic properties of diatomic metal borides in gas-phase experimentally ${ }^{4,5}$ and also to understand their bonding characteristics theoretically. ${ }^{6-10}$ Iridium is also an important metal catalyst in the formation of carbon-hydrogen and carbon-oxygen bonds. ${ }^{11,12}$ Iridium compounds' catalytic properties in chemical reactions are well known, but their spectroscopic properties are not ready available. Gas-phase diatomic iridium compounds with one main group element such as IrO (Refs. 13 and 14), IrN (Refs. 15-17), and IrC (Refs. 17-19) are the only ones that were characterized spectroscopically.

A very effective means of studying the bonding nature of small molecules is from an analysis of the optical spectra recorded at sufficiently high resolution to resolve not only the rotational structure but also the spin-fine and hyperfine structures. Rotational structure gives details on the bond length and, if available, the first lines of the rotational branches help to confirm the assignment of electronic state. ${ }^{20}$ The spin-fine and hyperfine structures provide information concerning electron occupation in different molecular orbitals (MOs). The magnetic hyperfine parameters measured ex-

\footnotetext{
${ }^{a)}$ Author to whom correspondence should be addressed. Telephone: (852) 2859 2155. Fax: (852) 2857 1586. Electronic mail: hrsccsc@hku.hk.
}

perimentally can be related to averages of various spin densities, of the unpaired valence electrons at the nuclei. ${ }^{21,22}$

In this paper, we report the analysis of an electronic transition system of the IrB molecule recorded using the technique of laser vaporization/reaction free jet expansion and laser induced fluorescence (LIF) spectroscopy. The electronic transition identified is assigned as the $[16.5]^{3} \Pi_{2}$ $-X^{3} \Delta_{3}$ system. The four $\operatorname{IrB}$ isotopic molecules are ${ }^{191}{ }^{1}{ }^{10} \mathrm{~B}$, ${ }^{193} \operatorname{Ir}^{10} \mathrm{~B},{ }^{191} \mathrm{Ir}^{11} \mathrm{~B}$, and ${ }^{193} \mathrm{Ir}^{11} \mathrm{~B}$ and their relative natural abundance are, respectively, 1.0, 1.68, 4.02, and 6.77. In our study, the spectra of ${ }^{191} \mathrm{Ir}^{11} \mathrm{~B}$ and ${ }^{193} \mathrm{Ir}^{11} \mathrm{~B}$ were recorded with good intensity; however, we could not resolve $\operatorname{Ir}^{10} \mathrm{~B}$ isotope transition lines except in the $(0,0)$ band. The $(2,0)$ band was found to be perturbed. Furthermore, hyperfine interaction arising from unpaired electrons and the magnetic moment of the boron atom with nuclear spin of $I=3 / 2$ have also been observed and analyzed.

\section{EXPERIMENT}

The electronic transition spectrum of IrB was obtained using a laser vaporization/reaction free jet expansion LIF spectrometer. Descriptions of the spectrometer and the laser ablation system were given in earlier publication. ${ }^{23}$ Only a brief description of the relevant experimental conditions for obtaining the $\mathrm{IrB}$ spectrum is given here. Pulses of laser radiation at $532 \mathrm{~nm}, 5 \mathrm{~mJ}$, and $10 \mathrm{~ns}$ from a Nd:YAG (yttrium aluminum garnet) laser were focused onto the surface of a iridium rod to generate iridium atoms. A pulsed valve, synchronized with appropriate delay time, released a gas mixture of $1 \% \mathrm{~B}_{2} \mathrm{H}_{6}$ in argon to react with the iridium atom to produce IrB. The Nd:YAG-pulsed valve system was operated at $10 \mathrm{~Hz}$. A diode-pumped solid-state laser with $6 \mathrm{~W}$ output power and at $532 \mathrm{~nm}$ was used to pump the ring dye laser operating with R560 and R6G dyes in the visible region, which was used to excite the jet cooled IrB molecules. LIF signal was collected by a lens system and detected by a photomultiplier tube. Linewidths of about $250 \mathrm{MHz}$ were 


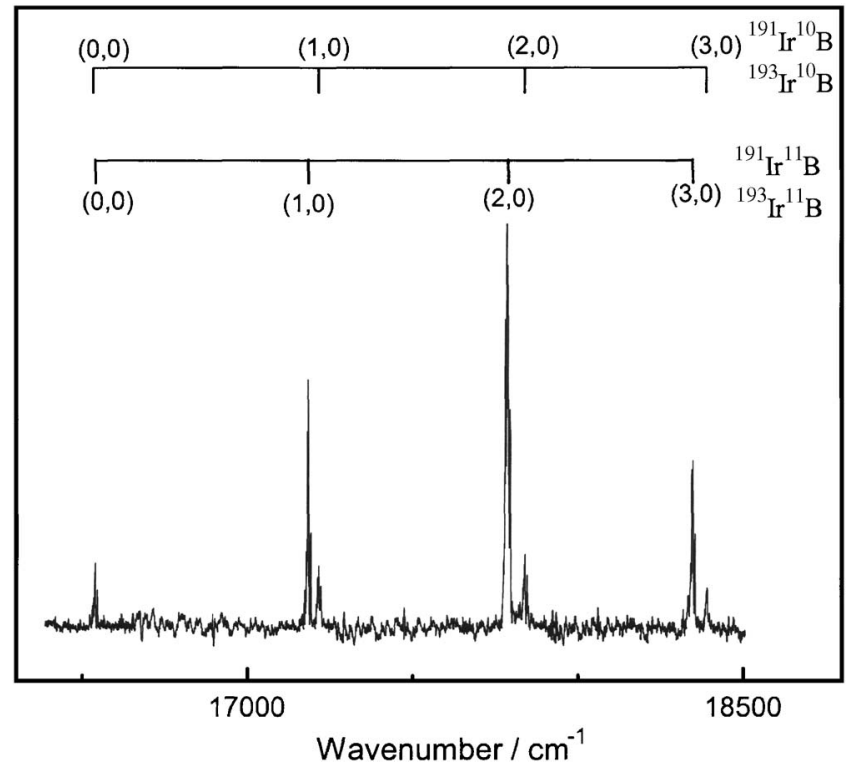

FIG. 1. Low-resolution LIF spectrum of the $[16.5]^{3} \Pi_{2}-X^{3} \Delta_{3}$ band system of IrB.

obtained. The wavelength of the dye laser was measured using a wavemeter with an accuracy of one part in $10^{7}$. The absolute accuracy of the measured line position is about $\pm 0.002 \mathrm{~cm}^{-1}$.

\section{RESULTS AND DISCUSSION}

\section{A. Low-resolution broadband spectrum}

Low-resolution LIF spectrum of IrB in the visible region between 16000 and $18500 \mathrm{~cm}^{-1}$ has been recorded. Figure 1 shows a broadband low-resolution scan of the IrB spectrum. There are two vibronic sequences belonging to the two isotopes: $\operatorname{Ir}^{10} \mathrm{~B}$ and $\operatorname{Ir}^{11} \mathrm{~B}$. With the exception of the $(0,0)$ band, we were able to resolve the transition bands due to the ${ }^{191} \mathrm{Ir}$ and ${ }^{193} \mathrm{Ir}$ isotopes. It is easily noticed that the $(2,0)$ band is a stronger transition. We have recorded high-resolution spectrum of the $(v, 0)$ bands with $v=0-3$.

\section{B. Assignment of the observed system}

Each of the recorded bands at high resolution shows resolved $P, Q$, and $R$ branches. Line assignment was straightforward. The $(0,0)$ band head was observed at $16547 \mathrm{~cm}^{-1}$,

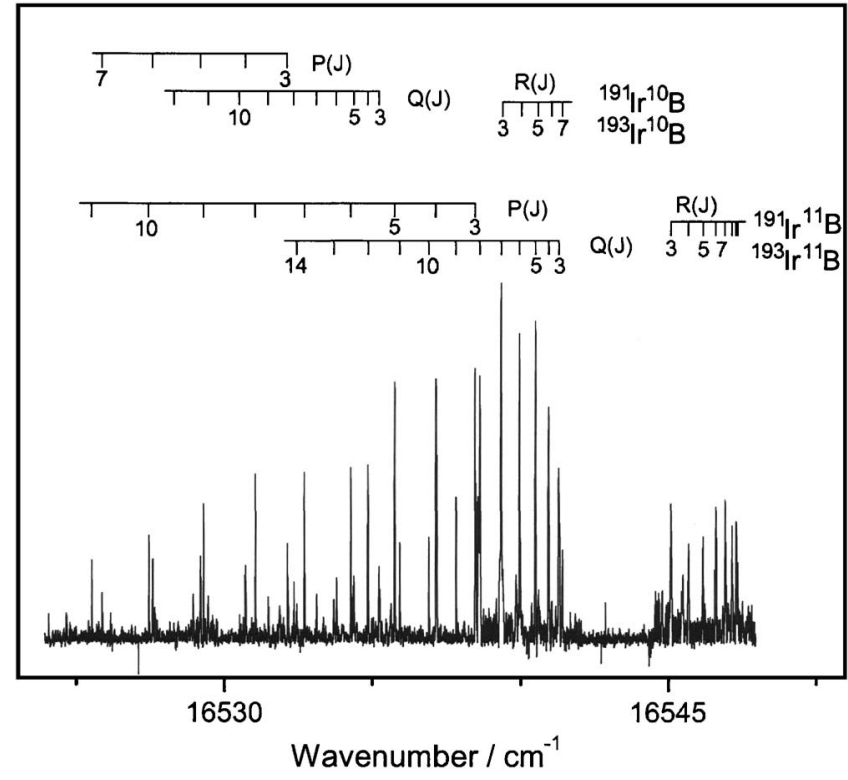

FIG. 2. The $(0,0)$ band of the $[16.5]^{3} \Pi_{2}-X^{3} \Delta_{3}$ transition of IrB.

which is shown in Fig. 2. We were able to resolve molecular transition lines with ${ }^{10} \mathrm{~B}$ and ${ }^{11} \mathrm{~B}$ atoms; the lines belonging to the $\operatorname{Ir}^{10} \mathrm{~B}$ isotopes are much weaker in intensity than those of the $\mathrm{Ir}^{11} \mathrm{~B}$. However, transition lines from the two iridium isotopes, ${ }^{191} \mathrm{Ir}$ and ${ }^{193} \mathrm{Ir}$, were not resolved in the $(0,0)$ band. The observation of the first lines, $P(3), Q(3)$, and $R(3)$ lines, established that this is a $\Omega^{\prime}=2$ and $\Omega^{\prime \prime}=3$ transition. The vibrational quantum number assignment was confirmed by examining the isotopic shift between the $\operatorname{Ir}^{10} \mathrm{~B}$ and $\operatorname{Ir}^{11} \mathrm{~B}$. In addition, the $R$ branch is relatively weaker than the $P$ and $Q$ branches, which is consistent with a $\Delta \Lambda=-1$ transition. Since only low $J$ lines $(J<17)$ were observed, the line positions of this band were fitted to the following expression:

$$
\begin{aligned}
\nu_{o}= & T_{o}+B^{\prime} J^{\prime}\left(J^{\prime}+1\right)-D^{\prime}\left[J^{\prime}\left(J^{\prime}+1\right)\right]^{2} \\
& -\left\{B^{\prime \prime} J^{\prime \prime}\left(J^{\prime \prime}+1\right)-D^{\prime \prime}\left[J^{\prime \prime}\left(J^{\prime \prime}+1\right)\right]^{2}\right\},
\end{aligned}
$$

where the ' and "refer to the upper and low states, respectively. Isotopic molecular transition lines of $\operatorname{Ir}^{10} \mathrm{~B}$ and $\operatorname{Ir}^{11} \mathrm{~B}$ were measured and fitted. The centrifugal constant $D$ was fixed at the calculated values using the Kratzer relation. ${ }^{20}$ Determined molecular constants of individual bands, equilibrium molecular constants, and bond lengths are given in

TABLE I. Molecular constants for the upper $[16.5]^{3} \Pi_{2}$ and the $X^{3} \Delta_{3}$ states of $\operatorname{IrB}\left(\mathrm{cm}^{-1}\right)$.

\begin{tabular}{lccccc}
\hline \hline State & Parameter & ${ }^{191} \operatorname{Ir}^{11} \mathrm{~B}$ & ${ }^{193} \operatorname{Ir}^{11} \mathrm{~B}$ & ${ }^{191} \mathrm{Ir}^{10} \mathrm{~B}$ & ${ }^{193} \operatorname{Ir}^{10} \mathrm{~B}$ \\
\hline$[16.5]^{3} \Pi_{2}$ & $T_{3}$ & $18350.286(2)$ & $18349.943(2)$ & & \\
& $B_{3}$ & $0.47235(3)$ & $0.47204(3)$ & & \\
& $T_{2}$ & $17791.129(2)$ & $17790.787(2)$ & & \\
& $B_{2}$ & $0.47455(2)$ & $0.47424(3)$ & & \\
& $T_{1}$ & $17186.970(2)$ & $17186.679(2)$ & & $16535.824(2)$ \\
& $B_{1}$ & $0.47321(2)$ & $0.47294(2)$ & & $0.51860(2)$ \\
& $T_{0}$ & $16541.845(1)$ & $16541.845(1)$ & $16535.824(2)$ & 0.000 \\
$X^{3} \Delta_{3}$ & $B_{0}$ & $0.47336(4)$ & $0.47336(4)$ & $0.51860(2)$ & 0.000 \\
& $T_{0}$ & 0.000 & 0.000 & $0.56684(2)$ \\
\hline \hline
\end{tabular}


TABLE II. Equilibrium molecular constants and bond length for the upper $[16.5]{ }^{3} \Pi_{2}$ and the $X^{3} \Delta_{3}$ states of IrB and the isotopes (values given in parentheses are one standard error in last significant figures quoted).

\begin{tabular}{|c|c|c|c|c|c|c|}
\hline \multirow[b]{2}{*}{ State } & \multirow[b]{2}{*}{ Parameter } & \multirow[b]{2}{*}{${ }^{193} \mathrm{Ir}^{11} \mathrm{~B}$} & \multicolumn{2}{|c|}{${ }^{191} \mathrm{Ir}^{11} \mathrm{~B}$} & \multicolumn{2}{|c|}{${ }^{193} \operatorname{Ir}^{10} \mathrm{~B}$} \\
\hline & & & Obs. & Calc. ${ }^{a}$ & Obs. & Calc. ${ }^{a}$ \\
\hline \multirow[t]{8}{*}[16.5]{$^{3} \Pi_{2}$} & $T_{e}$ & $16203.627(2)$ & $16203.415(2)$ & $\cdots$ & & \\
\hline & $\omega_{e}$ & $686.968(2)$ & $687.436(2)$ & 687.24 & & \\
\hline & $\omega_{e} X_{e}$ & $21.067(2)$ & $21.155(2)$ & 21.14 & & \\
\hline & $B_{e}$ & $0.473579(2)$ & 0.47385 & 0.47384 & & \\
\hline & $\alpha_{e}$ & 0.00043 & 0.00043 & 0.00043 & & \\
\hline & $r_{e}(\AA)$ & 1.8487 & & & & \\
\hline & $T_{0}$ & & & & 16535.824 & $\cdots$ \\
\hline & $B_{0}$ & & & & $0.51860(2)$ & 0.51792 \\
\hline \multirow{2}{*}{$X^{3} \Delta_{3}$} & $B_{0}$ & $0.51809(4)$ & $0.51809(4)$ & 0.51838 & $0.56684(2)$ & 0.56686 \\
\hline & $r_{0}(\AA)$ & 1.7675 & & & & \\
\hline
\end{tabular}

${ }^{a}$ Molecular constants were calculated using isotopic relationships and the values from ${ }^{193} \operatorname{Ir}^{11} \mathrm{~B}$.

Tables I and II. A list of the measured transition line positions of the observed $[16.5]^{3} \Pi_{2}-X^{3} \Delta_{3}$ system of $\operatorname{Ir}^{10} \mathrm{~B}$ and $\mathrm{Ir}^{11} \mathrm{~B}$ is available from EPAPS. ${ }^{24}$

\section{Perturbation at the $(2,0)$ band}

We noticed extra transition lines appearing in the spectrum of the $(2,0)$ band. Figure 3 shows a portion of the spectrum with the $P, Q$, and $R$ branches labeled and extra lines from the perturbing state are also marked. The transition lines of the $(2,0)$ band are broad and with unresolved hyperfine structure. The usual $P, Q$, and $R$ branches were easily identified and assigned. Extra lines that perturbed the $v=2$ level were also assigned. It is easily noted that the linewidth of the perturbing line is unusually large, which is due to the unresolved hyperfine structure and the overlapping of isotopic ${ }^{191} \mathrm{Ir}^{11} \mathrm{~B}$ and ${ }^{193} \mathrm{Ir}^{11} \mathrm{~B}$ molecular lines. Judging from the unresolved isotopic lines, we infer that the perturbing state has vibrational quantum number equal to 0 . The perturbing state was found to have $\Omega^{\prime}=3$ and $\Omega^{\prime \prime}=3$. Both the ${ }^{191} \mathrm{Ir}^{11} \mathrm{~B}$ and ${ }^{193} \mathrm{Ir}^{11} \mathrm{~B}$ lines were affected by the perturbing

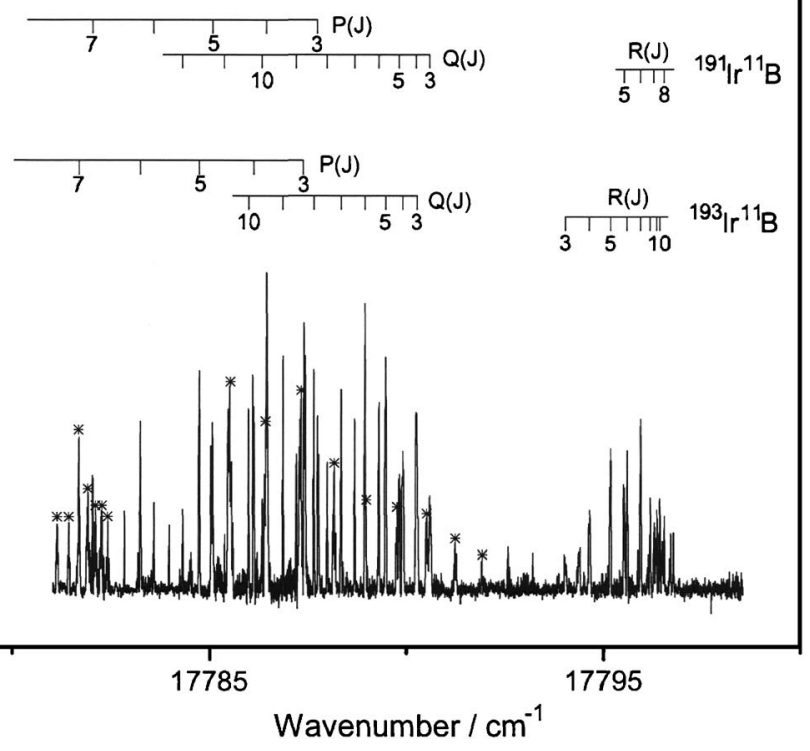

FIG. 3. The $(2,0)$ band of the $[16.5]^{3} \Pi_{2}-X^{3} \Delta_{3}$ transition of IrB. state. Term values of the upper states were obtained by adding the corresponding lower state term values to the transition frequencies. Figure 4 depicts a reduced term value plot of the $v=2$ level of the $[16.5]^{3} \Pi_{2}$ state and the $v=0$ of perturbing $\Omega^{\prime}=3$ level. If the data were fitted to expression (1) without taking into account of the perturbation, the $B_{2}$ value obtained was $0.47424 \mathrm{~cm}^{-1}$. However, assuming that no perturbation exists, the calculated $\mathrm{B}_{2}$ value using known $B$ values from other bands was $0.47250 \mathrm{~cm}^{-1}$. The increase of $0.00174 \mathrm{~cm}^{-1}$ in the rotational constant indicates that the $v$ $=2$ level has been "pushed" by the perturbing state. In addition, the band origin, $T_{2}$, was also pushed up by $0.13 \mathrm{~cm}^{-1}$ from the expected position.

\section{Electronic configuration and the electronic states}

Marr et al. ${ }^{17}$ discussed the MO energy level diagram for $\mathrm{IrC}$ and $\mathrm{IrN}$. Figure 5 shows a MO energy level diagram formed from the $6 s$ and $5 d$ atomic orbitals (AOs) of the iridium atom and the $2 p$ orbital of a main group element. The lower energy $1 \sigma$ and $1 \pi$ MOs and the higher energy $3 \sigma$ and $2 \pi$ MOs are formed from the main group $2 p \mathrm{AO}$ and iridium $5 d \sigma, 5 d \pi$, and $6 p \sigma$ and $6 p \pi$ AOs. The $2 \sigma \mathrm{MO}$ is essentially the iridium $6 s \mathrm{AO}$. The $1 \delta \mathrm{MO}$ is the iridium $5 d \delta \mathrm{AO}$, be-

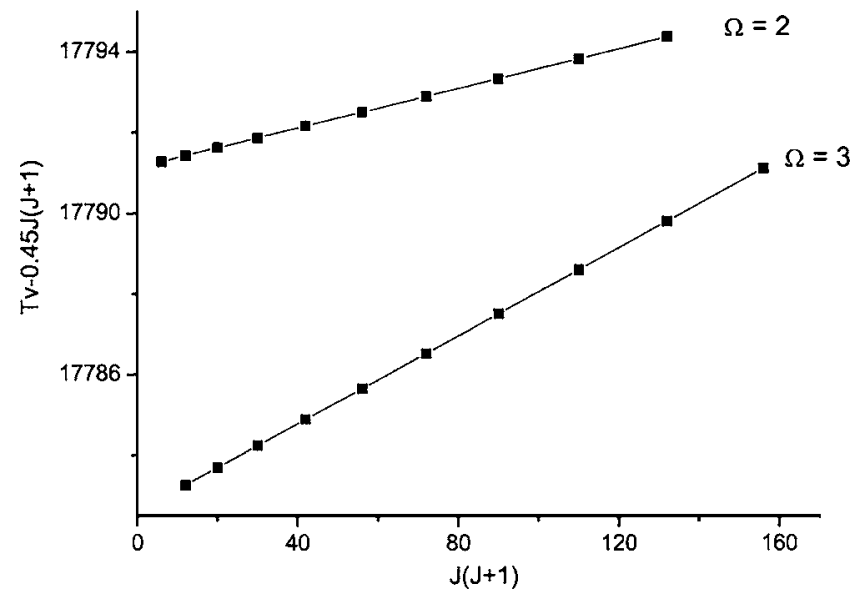

FIG. 4. Reduced term value plot of $v=2$ level of $\Omega=2$ and $\Omega=3$ states of IrB. 


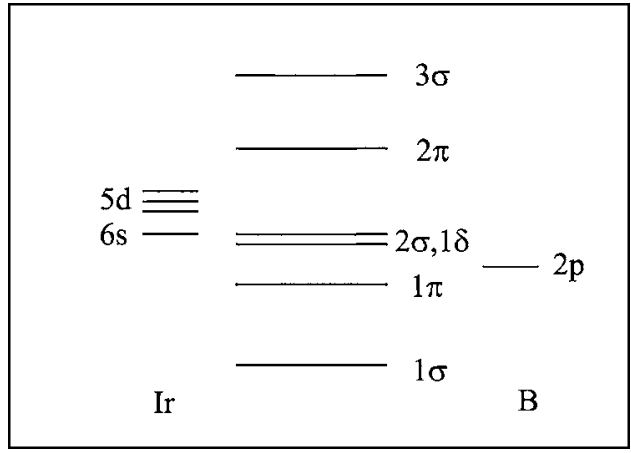

FIG. 5. MO energy level diagram of IrB.

cause there is no other $\delta$ symmetry orbital around. The ground state of the diatomic molecules formed from iridium and a main group element are

$$
\begin{aligned}
& \operatorname{IrC}\left(X^{2} \Delta_{5 / 2}\right): \quad(1 \sigma)^{2}(1 \pi)^{4}(2 \sigma)^{2}(1 \delta)^{3} \rightarrow{ }^{2} \Delta, \\
& \operatorname{IrN}\left(X^{1} \Sigma^{+}\right): \quad(1 \sigma)^{2}(1 \pi)^{4}(2 \sigma)^{2}(1 \delta)^{4} \rightarrow{ }^{1} \Sigma^{+} .
\end{aligned}
$$

If the trend of putting the electron into the MOs is consistent with the above two iridium compounds, then $\operatorname{IrB}$ would have two electrons in the $1 \delta \mathrm{MO}$ and the electronic configuration obtained is

$$
(1 \sigma)^{2}(1 \pi)^{4}(2 \sigma)^{2}(1 \delta)^{2} \rightarrow{ }^{1} \Sigma^{+},{ }^{3} \Sigma^{-}, \text {and }{ }^{1} \Gamma .
$$

From the first lines of various rotational branches, we concluded that the ground state has a spin component of $\Omega=3$. Close examination indicates that none of the electronic states in Eq. (4) has a spin component with $\Omega=3$; therefore, the lower state could not possibly arise from such an electronic configuration. Another possible low energy electronic configuration is

$$
\text { IrB: } \quad(1 \sigma)^{2}(1 \pi)^{4}(1 \delta)^{3}(2 \sigma)^{1} \rightarrow{ }^{1} \Delta \text { and }{ }^{3} \Delta_{i} .
$$

The difference between configurations (2) and (5) is that the two MOs, $1 \delta$ and $2 \sigma$, are so close in energy that in the case of IrB both are partially filled; however, in the $\operatorname{IrC}$, the extra electron goes to the $2 \sigma$ orbital giving the configuration (2). This energy order of the MOs is similar to that of rhodium carbide, nitride, oxide, and the fluoride molecules in Ref. 25. Due to the fact that the number of electrons in the $\delta$ orbital is more than one-half filled, the ${ }^{3} \Delta$ state arising from the above configuration is an inverted ${ }^{3} \Delta$ state. Such a ${ }^{3} \Delta_{i}$ state has $\Omega$ values equal to 1,2 , and 3 , and the $\Omega=3$ being the lowest energy. This fits well with the experimental observation and, therefore, is a reasonable assignment for the ground state. This electronic configuration is similar to that of the $\mathrm{FeC}$ molecule, which also has $X^{3} \Delta_{3}$ as the ground state. ${ }^{26}$ As for the other spin-orbit components of the ${ }^{3} \Delta_{i}$ ground state, the $1 \delta$ orbital is essentially the iridium $5 d \delta \mathrm{AO}$, and it is possible to obtain an estimate of the spin-orbit parameter for the $X^{3} \Delta$ state from the atomic value of iridium. Lefebvre-Brion and Field ${ }^{27}$ tabulated a value of $\zeta=3617 \mathrm{~cm}^{-1}$ for iridium atom. Since the first order energy expression for calculating the spin-orbit components is $A \Lambda \Sigma$, the separation between the

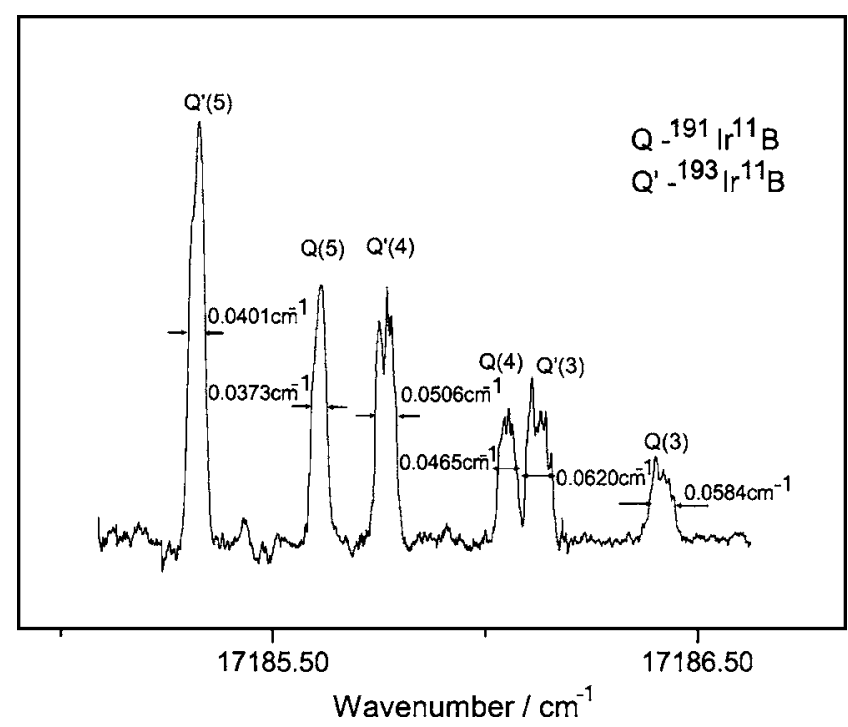

FIG. 6. The $(1,0)$ band of the $[16.5]^{3} \Pi_{2}-X^{3} \Delta_{3}$ transition of IrB with partially resolved hyperfine structure.

spin-orbit components is estimated to be very large and probably over $7000 \mathrm{~cm}^{-1}$. With our low temperature jet expansion source, it is expected that the higher energy spin-orbit components would not be appreciably populated. Nevertheless, with the origin of the $(0,0)$ band at $16541 \mathrm{~cm}^{-1}$, we searched within the energy range of our laser sources between 15000 and $18000 \mathrm{~cm}^{-1}$ to look for other electronic transitions or transitions from other spin-orbit components of the ground state, but none was found. As for the assignment of the upper state, our experience is that a relatively strong transition would normally have $\Delta S=0$, when combining with the selection rule of $\Delta \Lambda=-1$ with $\Omega^{\prime}=2$, it is reasonable that the upper state is assigned to be ${ }^{3} \Pi_{2}$. Considering the promotion of an electron from the $2 \sigma$ to the $2 \pi \mathrm{MO}$, the following electronic configuration is obtained:

$$
(1 \sigma)^{2}(1 \pi)^{4}(1 \delta)^{3}(2 \pi)^{1} \rightarrow{ }^{1} \Pi,{ }^{3} \Pi_{i},{ }^{1} \Phi, \text { and }{ }^{3} \Phi_{i} .
$$

The electronic configuration in Eq. (6) gives rise to four excited states of which only the ${ }^{3} \Pi_{i}$ and ${ }^{3} \Phi_{i}$ have spin-orbit components with $\Omega=2$, but only the ${ }^{3} \Pi_{i}$ state would satisfy the selection rule of $\Delta \Lambda=-1$. We have therefore assigned the observed transition as $[16.5]^{3} \Pi_{2}-X^{3} \Delta_{3}$ system. In view of the fact that the spin-orbit interaction is very large, it might be also appropriate to use Hund's case (c) notation to describe the electronic states, in which case the label would be just [16.5] 2.

\section{E. Hyperfine structure in the observed system}

Figure 6 shows the band head region of the $(1,0)$ band of the $[16.5]^{3} \Pi_{2}-X^{3} \Delta_{3}$ transition of the ${ }^{191}{ }^{1}{ }^{11} \mathrm{~B}$ and ${ }^{193} \mathrm{Ir}{ }^{11} \mathrm{~B}$. It is easily noticed that the transition lines have linewidth wider than the expected Doppler width and with partially resolved structure. The unusually large linewidth basically arises from unresolved hyperfine structure, which is due to the interaction between the magnetic moment of unpaired electrons and the magnetic moment of nucleus in the 
molecule. Since both ${ }^{191} \operatorname{Ir}\left(I=3 / 2, \mu / \mu_{N}=0.146\right)$ and ${ }^{193} \mathrm{Ir}$ $\left(I=3 / 2, \mu / \mu_{N}=0.159\right)$ atoms have small nuclear magnetic moment and the ${ }^{11} \mathrm{~B}\left(I=3 / 2, \mu / \mu_{N}=2.689\right)$ atom has a much larger nuclear magnetic moment, ${ }^{28}$ it is expected that the hyperfine structure should be dominated by the ${ }^{11} \mathrm{~B}$ atom. With only partially resolved hyperfine structure, it is not possible to obtain information concerning the small hyperfine interaction from the Ir atoms. As shown in Fig. 6, the rapid decrease in linewidth as $J$ value increases indicates that the hyperfine coupling case conforms to Hund's case $\left(a_{\beta}\right)$ coupling scheme. $^{22}$

The hyperfine Hamiltonian responsible for the magnetic interactions in a diatomic molecule is

$$
\hat{H}_{\mathrm{hfs}}=a I_{z} L_{z}+b \hat{I} \cdot \hat{S}+c I_{z} S_{z},
$$

where $a, b$, and $c$ parameters are the same as those defined by Frosch and Foley. ${ }^{29}$ The three terms in the hyperfine Hamiltonian are, respectively, the nuclear spin-orbit, the Fermi contact, and the dipolar electron spin and nuclear spin interactions. For $\Pi$ and $\Delta$ states with large spin-orbit interaction, the angular momentum coupling case is appropriately described by Hund's coupling case $\left(a_{\beta}\right)$. In such a coupling case, the grand total quantum number $F$ results from the coupling of the nuclear spin $I$ with angular momentum $J$, where $F=I+J$. Hyperfine matrix elements of a $\Pi$ and a $\Delta$ state in Hund's coupling case $\left(a_{\beta}\right)$ can be found in Ref. 30. The matrix elements important for this analysis are

$$
\begin{aligned}
& \left\langle J \Omega I F\left|\hat{H}_{\mathrm{hfs}}\right| J \Omega I F\right\rangle=\frac{\Omega \cdot h[F(F+1)-I(I+1)-J(J+1)]}{2 J(J+1)}, \\
& \left\langle J \Omega I F\left|\hat{H}_{\mathrm{hfs}}\right| J-1, \Omega I F\right\rangle=\frac{h \cdot \sqrt{J^{2}-\Omega^{2}} \sqrt{(J+I+F+1)(F+J-I)(J+I-F)(F+I-J+1)}}{2 J \sqrt{2 J+1)(2 J-1)}},
\end{aligned}
$$

where $h=\Lambda \cdot a+(b+c) \cdot \Sigma$. For the upper ${ }^{3} \Pi_{2}(\Lambda=1$ and $\Sigma$ =1) state and the ground ${ }^{3} \Delta_{3}(\Lambda=2$ and $\Sigma=1)$ state, the $h^{\prime}$ and $h^{\prime \prime}$ are $a+b+c$ and $2 a+b+c$, respectively. We used the linewidth (FWHM, full width at half maximum) of the hyperfine lines and the diagonal matrix elements only [Eq. (8)] to obtain an estimate of the hyperfine parameters. As shown in Fig. 6, the FWHM linewidth of $Q(3)$ and $P(3)$ lines were, respectively, measured to be $0.0584 \mathrm{~cm}^{-1}$ and $0.0426 \mathrm{~cm}^{-1}$, from which the hyperfine parameters for the upper and the lower states were estimated to be $h^{\prime}=0.012 \mathrm{~cm}^{-1}$ and $h^{\prime \prime}$ $=0.029 \mathrm{~cm}^{-1}$. The magnetic hyperfine parameter $h$ is related to the expectation values of the coordinates of electron near the spinning nucleus ${ }^{31}$ and can be expressed as averages over electronic coordinates and is listed as follows (in $\mathrm{cm}^{-1}$ unit):

$$
\begin{aligned}
& a=\left(\frac{\mu_{\mathrm{o}}}{4 \pi h c}\right) g g_{N} \mu_{B} \mu_{N} \sum_{i}\left\langle r_{i}^{-3}\right\rangle \\
& b_{F}=\left(\frac{\mu_{\mathrm{o}}}{4 \pi h c}\right) \frac{8 \pi}{3} g g_{N} \mu_{B} \mu_{N}\left(\frac{1}{2 S}\right) \sum_{i}\left\langle\psi_{i}^{2}(0)\right\rangle \\
& c=\left(\frac{\mu_{\mathrm{o}}}{4 \pi h c}\right) \frac{3}{2} g g_{N} \mu_{B} \mu_{N}\left(\frac{1}{2 S}\right) \sum_{i}\left\langle 3 \cos ^{2} \theta_{2}-1\right\rangle\left\langle r_{i}^{-3}\right\rangle
\end{aligned}
$$

$$
b=b_{F}-\frac{1}{3} c .
$$

A method of obtaining estimates of the hyperfine parameters is using $a b$ initio results for the atom. The $a, b$, and $c$ parameters can be estimated using the expressions in Eqs. (10)-(13) and the values of $\left\langle r_{i}^{-3}\right\rangle\left\langle r_{i}^{-3}\right\rangle^{24},\left\langle\psi_{i}^{2}(0)\right\rangle$, and $\left\langle 3 \cos ^{2} \theta_{i}-1\right\rangle$ from Morton and Preston. ${ }^{32}$ We obtained $a$ $=0.005 \mathrm{~cm}^{-1}, b=0.043 \mathrm{~cm}^{-1}$, and $c=-0.002 \mathrm{~cm}^{-1}$. The calculated $h^{\prime}$ and $h^{\prime \prime}$ are, respectively, 0.046 and $0.051 \mathrm{~cm}^{-1}$, which are larger than those values determined experimentally by almost a factor of 2 . This discrepancy could be due to the fact that the estimated $h^{\prime}$ and $h^{\prime \prime}$ values from experimental data are only preliminary and have relatively large errors because broad overall linewidths were measured. In addition, there have not been any theoretical calculations to estimate the contributions of various AOs to the MOs responsible for the hyperfine structure. Moreover, the effect of the nuclear quadrupole interaction, $e^{2} Q q$, has not been included in both experimental and theoretical treatments. A high resolution and detailed study of the hyperfine structure arising from both nuclei is important to resolve the noted discrepancy.

In summary, we report the first observation of an electronic transition spectrum of the $\mathrm{IrB}$ molecule. The observed transition has been assigned as the $[16.5]^{3} \Pi_{2}-X^{3} \Delta_{3}$ transition. The bond length of the lower state has been determined to be $1.7675 \AA$. The hyperfine parameters obtained also provide reasonable support to the assignment of the electronic states in this transition.

\section{ACKNOWLEDGMENTS}

The work described here was supported by a grant from the Research Grants Council of the Hong Kong Special Administrative Region, China (Project No. HKU 7105/04P). We would like to thank the referee for the discussion concerning the assignment of the observed transition and critically reading the manuscript. 
${ }^{1}$ M. Trenary, in Materials Science of Carbides, Nitrides and Borides, NATO Science Series Vol. 68, edited by Y. G. Gogotsi and R. A. Andrievski (Kluwer Academic, Dordrecht, 1998).

${ }^{2}$ B. Ganem and J. O. Osby, Chem. Rev. (Washington, D.C.) 86, 163 (1986)

${ }^{3}$ H. J. Choi, D. Roundy, H. Sun, M. L. Cohen, and S. G. Louie, Nature (London) 418, 758 (2002).

${ }^{4}$ P. K. Chowdhury and W. J. Balfour, J. Chem. Phys. 124, 216101 (2006).

${ }^{5}$ C. R. Brazier, J. I. Ruiz, and S. V. Parks, J. Mol. Spectrosc. 241, 1 (2007).

${ }^{6}$ C. W. Bauschlicher, Jr., and S. R. Langhoff, J. Chem. Phys. 101, 80 (1994)

${ }^{7}$ F. R. Ornellas and A. R. S. Valentim, J. Phys. Chem. 98, 12570 (1994).

${ }^{8}$ A. Ricca and C. W. Bauschlicher, Jr., Chem. Phys. Lett. 241, 241 (1995).

${ }^{9}$ M. Pelegrini, O. Roberto-Neto, and F. B. C. Machado, Int. J. Quantum Chem. 95, 205 (2003).

${ }^{10}$ I. Černušák, M. Dallos, H. Lischka, T. Müller, and M. Uhlár, J. Chem. Phys. 126, 214311 (2007).

${ }^{11}$ M. A. Ciriano, L. A. Oro, and M. T. Camellini, Organometallics 14, 4764 (1995).

${ }^{12}$ R. Jimenez-Catafio and M. B. Hall, Organometallics 15, 1889 (1996).

${ }^{13}$ V. Raziunas, G. Macur, and S. Katz, J. Chem. Phys. 43, 1010 (1965).

${ }^{14}$ K. Jansson and R. Scullman, J. Mol. Spectrosc. 43, 208 (1972).

${ }^{15}$ T. C. Steimle, A. J. Marr, S. A. Beaton, and J. M. Brown, J. Chem. Phys. 106, 2073 (1997).

${ }^{16}$ R. S. Ram, J. Lievin, and P. F. Bernath, J. Mol. Spectrosc. 197, 133 (1999).

${ }^{17}$ A. J. Marr, M. E. Flores, and T. C. Steimle, J. Chem. Phys. 104, 8183 (1996).

${ }^{18}$ K. Jansson and R. Scullman, J. Mol. Spectrosc. 36, 246 (1970).
${ }^{19}$ T. Ma, J. W.-H. Leung, and A. S.-C. Cheung, Chem. Phys. Lett. 385, 259 (2004).

${ }^{20}$ G. Herzberg, Spectra of Diatomic Molecules (Van Nostrand, New York, 1950).

${ }^{21}$ H. Lefebvre-Brion and R. W. Field, The Spectra and Dynamics of Diatomic Molecules (Elsevier, New York, 2004).

${ }^{22}$ T. Dunn, in Molecular Spectroscopy: Modern Research, edited by K. N. Rao, (Academic, New York, 1972), Vol. III, Chap. 4.4.

${ }^{23}$ Q. Ran, W. S. Tam, C. Ma, and A. S.-C. Cheung, J. Mol. Spectrosc. 198, 175 (1999).

${ }^{24}$ See EPAPS Document No. E-JCPSA6-128-011815 for the observed line positions of the $[16.5]^{3} \Pi_{2}-X^{3} \Delta_{3}$ transition. For more information on EPAPS, see http://www.aip.org/pubservs/epaps.html.

${ }^{25}$ R. Li, R. H. Jensen, W. J. Balfour, S. A. Shepard, and A. G. Adam, J. Chem. Phys. 121, 2591 (2004).

${ }^{26}$ W. J. Balfour, J. Cao, C. V. V. Prasad, and C. X. W. Qian, J. Chem. Phys. 103, 4046 (1995).

${ }^{27} \mathrm{H}$. Lefebvre-Brion and R. W. Field, Perturbations in the Spectra of Diatomic Molecules (Academic, New York, 1986).

${ }^{28}$ I. Mills, T. Cvitaš, K. Homann, N. Kallay, and K. Kuchitsu, Quantities, Units and Symbols in Physical Chemistry (Blackwell Scientific, London, 1993).

${ }^{29}$ R. A. Frosch and H. M. Foley, Phys. Rev. 88, 1337 (1952).

${ }^{30}$ Y. Azuma, J. A. Barry, M. P. J. Lyne, A. J. Merer, J. O. Schroder, and J. L. Femenias, J. Chem. Phys. 91, 1 (1989).

${ }^{31}$ C. H. Townes and A. L. Schawlow, Microwave Spectroscopy (Dover, New York, 1975).

${ }^{32}$ J. R. Morton and K. F. Preston, J. Magn. Reson. (1969-1992) 30, 577 (1978). 